\title{
Heat treatment of chlorinated waste in a rotating kiln: Problems with intermediate reaction products and solutions applied
}

\author{
F. Lemort ${ }^{\mathrm{a}, *}$, Y. Soudais ${ }^{\mathrm{b}}$, L. Moga $^{\mathrm{b}}$, Y. Blazeck ${ }^{\mathrm{a}, \mathrm{b}}$ \\ ${ }^{a}$ CEA Valrhô-Marcoule, Laboratoire des Procédés Innovants de Conditionnement (LPIC), BP 17171, \\ 30207 Bagnols-sur-Cèze Cedex, France \\ ${ }^{\mathrm{b}}$ UMR CNRS 2392, Laboratoire de Génie des Procédés des Solides Divisés, École des Mines d'Albi-Carmaux, \\ Campus Jarlard, 81013 Albi, France
}

\begin{abstract}
At the CEA, the incineration of radioactive wastes is carried out thanks to the IRIS process involving a oxidizing pyrolysis step performed with a rotary kiln. If the running of the process is very satisfactory for processing waste of a given composition, it appears that the behavior of the kiln may diverge when the composition of the waste change. Through the example of wastes containing a large amount of PVC and thanks to a very simple modelling of the heating of the kiln, it has been demonstrated that the deposits are involved in overheating of the pyrolysis kiln. Fundamental investigation carried out about the thermal degradation of PVC and cellulose have shown that the formation of intermediate PVC decomposition compounds together with the residues coming from the decomposition of the cellulose could be responsible of the large amount of deposits. In order to avoid the malfunctions it has been proposed to revise the furnace operating parameters by specifying a waste feed sequence that ensure a relaxation period to the furnace. By this way, the running of the facility shows a very good stability with a very thin temperature oscillation that can easily be explained by a thermal modelling of the kiln.
\end{abstract}

Keywords: Incineration process; Rotary kiln; Modelling; PVC decomposition

\section{Introduction}

High-temperature processing of organic solid waste is often used to decrease the waste volume and neutralize its inorganic content by oxidation. Among the various processes available, the CEA selected incineration in a rotating kiln as the most suitable to meet the requirements arising from contamination of the material by radioelements, all of which must be recovered from the ashes with very low-carbon content. The IRIS

\footnotetext{
* Corresponding author.

E-mail address: florent.lemort@cea.fr (F. Lemort).
} 
two-step process [1] fully meets these requirements. It comprises an oxidizing pyrolysis step during which chlorine is released at moderate temperature to minimize corrosion problems, and a calcining step to eliminate virtually all the carbon. However, although satisfactory results have been obtained for processing waste of a given composition, the behavior of the kiln may diverge when the composition changes. The malfunctions occur mainly in the first pyrolysis step where very high temperatures are recorded and plugging results from deposits on the kiln walls.

The work described in this article was undertaken to demonstrate that the deposits are involved in overheating of the pyrolysis kiln, and to account for their presence by the formation of intermediate PVC decomposition compounds. Revising the furnace operating parameters by specifying a waste feed sequence significantly improved the operation of the unit.

\section{Malfunctioning of a pyrolysis kiln}

\subsection{The IRIS process}

The IRIS process is intended for heat treatment of chlorinated organic waste contaminated by radioelements. It uses two rotating kilns: the first ensures oxidizing pyrolysis at $550{ }^{\circ} \mathrm{C}$ to release the chlorine at moderate temperature in order to limit corrosion phenomena, and is followed by calcining at $900^{\circ} \mathrm{C}$ to produce low-carbon ash $(<1 \mathrm{wt} \%)$ that can subsequently be processed to recover selected radioelements or vitrified for ultimate disposal. The off-gas arising from both steps is ducted to an afterburner to oxidize all the volatile compounds, then to a processing unit for HEPA filtration and gas scrubbing.

\subsection{Processing waste of variable composition}

The process was developed to deal with a very specific waste category comprising nearly $50 \% \mathrm{PVC}$. This composition, " $\mathrm{A}$ " in Table 1, is representative of waste mixtures arising from the glove boxes. While this type of waste can be processed without difficulty, it appears that some composition changes can result in malfunctions characterized unexpected temperature and pressure increases in the pyrolysis kiln. For example, waste "B" described in Table 1, containing 45\% cellulose and 25\% PVC, produces this type of phenomenon and requires that the process be interrupted for safety reasons.

This article focuses only on the pyrolysis furnace, a rotating kiln $200 \mathrm{~mm}$ in diameter heated over a length of $1200 \mathrm{~mm}$. The kiln rotates at $6 \mathrm{rpm}$ and is inclined at a $4 \%$ slope; the material residence time in the tube is about $5 \mathrm{~min}$. The Hastelloy $\mathrm{C} 276$ tube is divided into three heating zones in which the temperature is recorded at the upper and lower parts. For each zone, two thermocouples have been implemented under and above the kiln (Fig. 2). In normal operation waste is fed at $4 \mathrm{~kg} / \mathrm{h}$ to the pyrolysis kiln, in which the process is maintained in slightly oxidizing atmosphere $\left(5 \mathrm{Nm}^{3} / \mathrm{h} \mathrm{N}_{2}-1 \mathrm{Nm}^{3} / \mathrm{h} \mathrm{O}\right.$ ) flowing in the same direction than the waste (co-current reactor). Oxygen is supplied to burn the volatile tars and prevent them from forming deposits between the pyrolysis kiln and the afterburner.

Fig. 1 is a temperature record for all the zones in the oxidizing pyrolysis kiln during continuous processing over a 50 -h period. It is interesting to note that the temperatures in zones 2 and 3 vary successively; the differences are due to the different heat loads and to convection phenomena between the upper and lower ends. The temperature decreases at the lower end of zone 1 as cold waste is supplied to the kiln and is melted by

Table 1

Composition ( $\left.w \mathrm{wt}^{\%} \mathrm{)}\right)$ of two types of waste

\begin{tabular}{|c|c|c|}
\hline Waste & $A$ & $B$ \\
\hline Cotton & 5 & 14 \\
\hline Cleansing tissue & 5 & 41 \\
\hline Polyethylene & 5 & 4 \\
\hline Neoprene & 17.5 & 5.3 \\
\hline Latex & 17.5 & 10.7 \\
\hline PVC & 51 & 25 \\
\hline
\end{tabular}




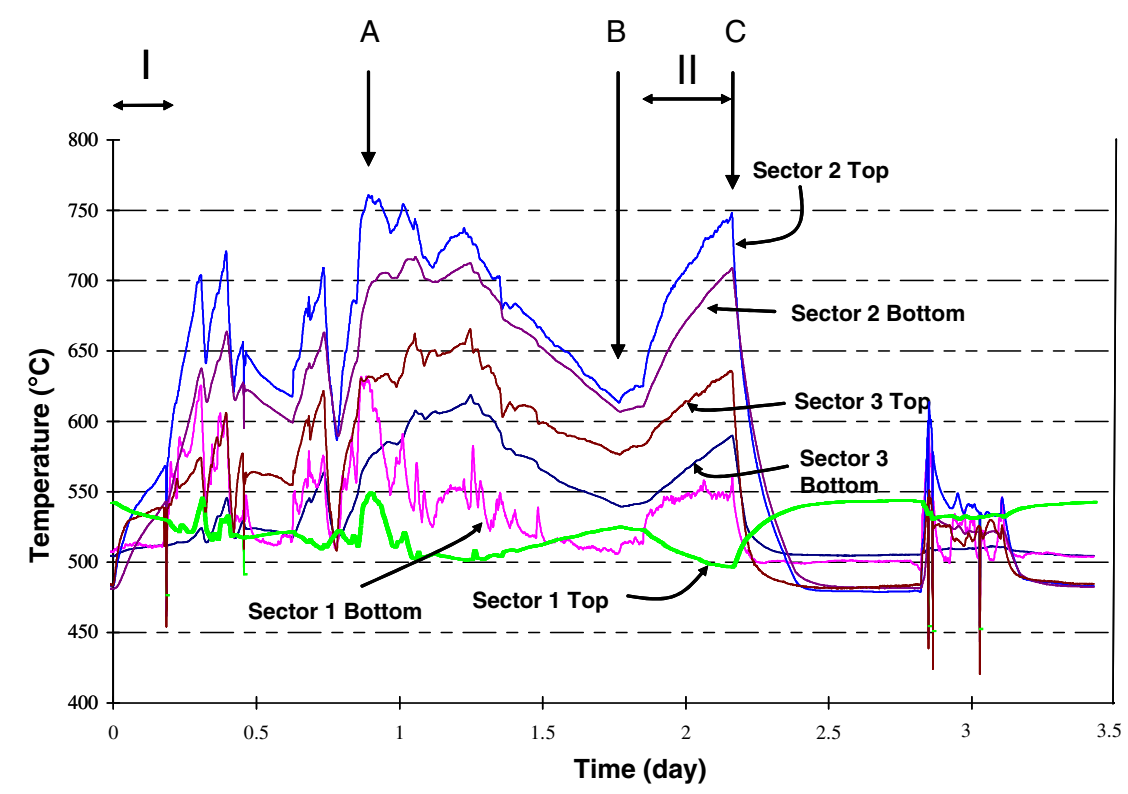

Fig. 1. Temperature variations in an oxidizing pyrolysis kiln during processing over a 2.5-day period.

absorbing heat. As the phenomena recorded in the upper portion of zone 2 are the most representative, this is the only temperature record used in the remainder of this article to describe the phenomena observed.

The curve for the upper portion of zone 2 shows a very significant temperature rise from $500{ }^{\circ} \mathrm{C}$ to $750{ }^{\circ} \mathrm{C}$ in one day. Shutting off the oxygen supply at point $A$ caused the temperature to diminish regularly to $620^{\circ} \mathrm{C}$. Resupplying oxygen at point $B$ after about $40 \mathrm{~h}$ again resulted in a sudden temperature rise. Interrupting the waste feed at point $C$ then caused a sudden temperature drop to about $500{ }^{\circ} \mathrm{C}$.

Monitoring the pressures in the unit shows major variations with periodic overpressure values that are undesirable for the safety of the process.

\subsection{Analysis of the observed phenomena}

It is a complex undertaking to interpret the curves shown in Fig. 1. However, zones I and II are of particular interest in that they exhibit the characteristic appearance of controlled temperature rises. Fig. 2 very schematically shows the heat balance of the rotating kiln in which the power injected, $Q \mathrm{~d} t$, is partially absorbed by the structure with a constant overall heat capacity $C$, and partially emitted in the off-gas and in the structure with an overall dissipation coefficient $K$.

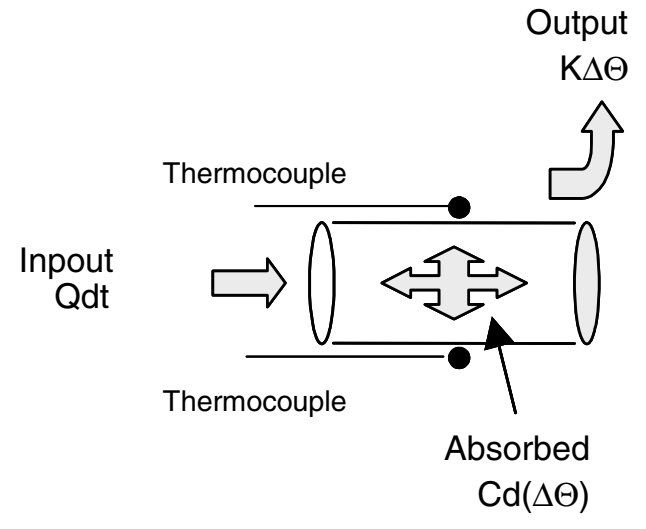

Fig. 2. Heat balance for calculation. 
$\Delta \Theta$ indicates the temperature difference between the system and the surrounding environment. The heat balance is the following:

$$
Q \mathrm{~d} t=K \Delta \Theta \mathrm{d} t+C \mathrm{~d}(\Delta \Theta)
$$

The temperature variation is thus theoretically described by the differential equation:

$$
\frac{\mathrm{d}(\Delta \Theta)}{\mathrm{d} t}+\frac{K}{C} \Delta \Theta=\frac{Q}{C}
$$

Solving this type of equation yields a mathematical representation of the temperature variation:

$$
\Theta(t)=\Theta_{0}+\frac{Q}{K}\left(1-\mathrm{e}^{-\frac{K}{C} t}\right)
$$

A parameter analysis was performed for zones I and II. Zone I corresponds to the beginning of the processing period, i.e. to the temperature rise due to loading the waste mixture in an empty furnace. The following parameters were determined:

$$
Q_{1} / K_{1}=51 \text { and } K_{1} / C_{1}=30
$$

The results of a simulation based on these parameters are shown in Fig. 3. Note the very satisfactory fit of the two curves.

Zone II corresponds to the temperature rise due to resupplying oxygen to the kiln. The following parameters were determined for the rising temperature curve:

$$
Q_{2} / K_{2}=169 \text { and } K_{2} / C_{2}=2.8
$$

The two results are significantly different, due to the variation of $C$ and $K$ during the test. The heat load in the furnace, $Q$ is about constant since the heat flow rate did not change between the two test periods. Then, results lead to calculate $C_{2} \approx 3 C_{1}$ showing that the thermal capacity of the furnace sensibly increased. This can be connected to the formation of a carbonaceous deposit during the test. The increase of the thermal capacity is coupled with a drop in the dissipation coefficient $K$ : the measured value of $K_{2} / K_{1}$ was 0.3 . This implies that the appearance of the deposit in the rotating tube diminishes the dissipation coefficient and thus increases the rate of the temperature rise. Actually, a sampling allowed to recover a deposit containing around $70 \%$ of carbon and other compounds such as zinc or phosphorus (present in initial wastes).

The following parameters were determined for the falling temperature curve:

$$
Q_{3} / K_{3}=-640 \text { and } K_{3} / C_{3}=2.8
$$

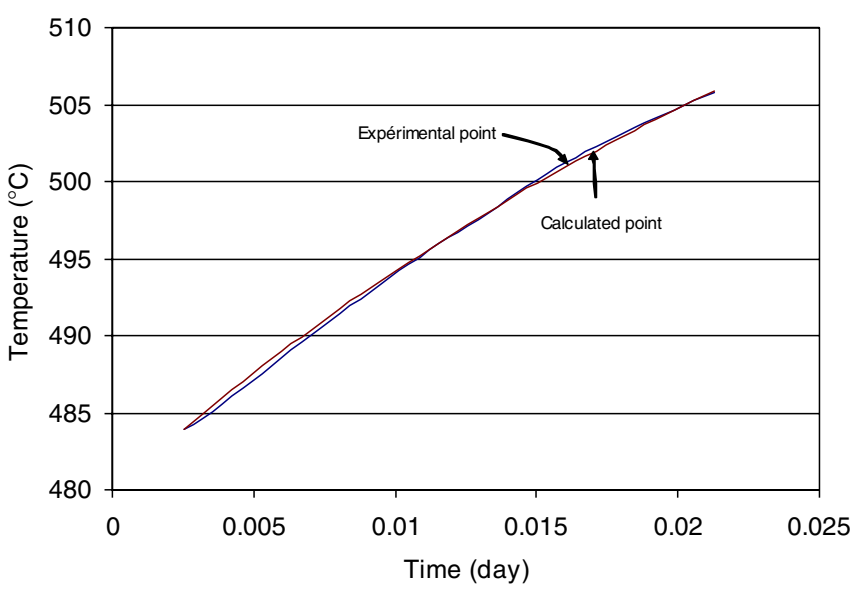

Fig. 3. Theoretical approach to overheating measured in the pyrolysis kiln. 


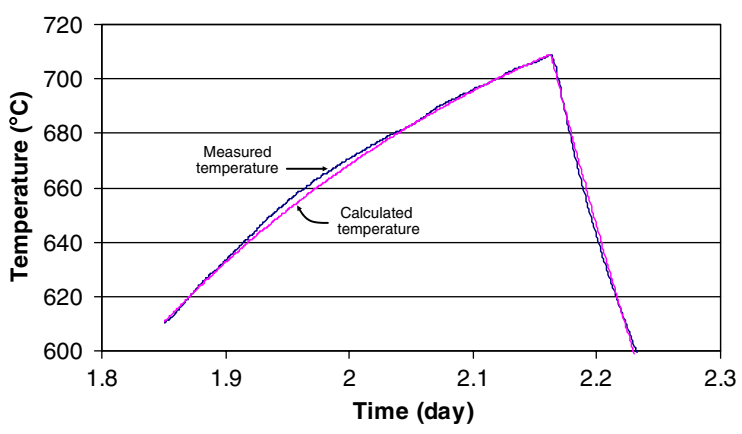

Fig. 4. Theoretical approach to overheating in the pyrolysis kiln after restoring the oxygen supply (rising curve) then interrupting the waste feed (falling curve).

The heat load term becomes negative, indicating that the heat supplied by combustion of the deposit is not sufficient to maintain the furnace temperature and is compensated by heat transport via the off-gas and by dissipation in the structure. The $K_{3} / C$ term is identical with the previously determined value.

The results of a simulation based on these parameters are shown in Fig. 4. Note the very satisfactory fit of the two curves.

The results cannot be analyzed in greater detail due to a lack of data concerning the heat capacity and to the fact that the power supplied to the kiln quickly returns to its maximum value after the feed stream is interrupted, making it impossible to determine the power dissipated by combustion of the carbonaceous layer inside the structure.

\subsection{Conclusion}

The results of the tests performed in an industrial-scale kiln show that it is very difficulty to maintain the stability of a rotating kiln supplied with organic waste with a composition that varies with respect to the design basis composition. Rapid temperature rises combined with major pressure variations make the process unstable and difficult to control. These conditions are mainly attributable to the appearance of significant deposits that must be eliminated.

\section{Investigation of pyrolytic decomposition of PVC and cellulose}

Basic studies were carried out to identify the decomposition mechanisms of some of the compounds present in the waste feed stream. They involved thermogravimetric analysis, and the results were interpreted using existing degradation models.

\subsection{Material}

All samples in the present study (PVC, cellulose) were pure polymers from Aldrich Chemical Company.

\subsection{Thermogravimetric and online FTIR analysis}

The samples were placed in the platinum crucible of the thermobalance. Heating rates ranging from 1 to $30{ }^{\circ} \mathrm{C} \mathrm{min}{ }^{-1}$ were applied $\left(5,10,20,30^{\circ} \mathrm{C} \min ^{-1}\right.$ for PVC; and $10,30{ }^{\circ} \mathrm{C} \mathrm{min}^{-1}$ for cellulose) and duplicate runs were carried out at all heating rates. Pyrolysis was carried out in $\mathrm{N}_{2}$ atmosphere, with the gas flow maintained at $27 \mathrm{~cm}^{3} \cdot \mathrm{min}^{-1}$. A Setaram model TGA 92-16 thermobalance was connected to the FTIR spectrophotometer by means of a heated line to allow continuous recordings of the IR spectra corresponding to the evolved gases. The spectrophotometer was a Perkin-Elmer System 2000 equipped with a gas cell and a DTGS detector. The heated line and gas-cell were temperature-regulated and maintained at $250{ }^{\circ} \mathrm{C}$ to prevent unwanted condensation and guarantee the most quantitative transfer possible of gaseous decomposition products and high detection sensitivity. 


\subsection{Results and discussion}

For all the TGA analysis curves the initial and final decomposition temperatures were variable, and the temperature increased with the heating rate, as expected.

\subsubsection{Interpretation of experimental curves}

3.3.1.1. PVC. Table 2 shows the temperatures of the DTG peaks (maximum degradation rate) observed during pyrolysis of PVC. The shape of the thermogram indicates three weight losses.

Note that the thermogram indicates only 2 peaks for a heating rate of $30^{\circ} \mathrm{C} / \mathrm{min}$ (Fig. 5); at a fast heating rate the degradations are recorded simultaneously and the peaks are superimposed.

3.3.1.2. Cellulose. Only heating rates of $10^{\circ} \mathrm{C} / \mathrm{min}$ and $30^{\circ} \mathrm{C} / \mathrm{min}$ were tested for cellulose. The thermogram indicates a single weight loss (Fig. 6, Table 3).

3.3.2. Kinetic model and data analysis

3.3.2.1. PVC. We tested one of the models proposed by Marcilla and Beltran [2]:

$$
\begin{aligned}
& \mathrm{PVC}^{k 1^{\prime}} \rightarrow a \mathrm{HCL}+b \mathrm{PVC}^{*} \\
& b \mathrm{PVC}^{*} \stackrel{k 2^{\prime}}{\rightarrow} c \mathrm{G}+e \mathrm{RS} \\
& e \mathrm{RS}^{k 3^{\prime}} \rightarrow f \mathrm{G}^{\prime}+g \mathrm{RS}^{*}
\end{aligned}
$$

The kinetic equations are the following:

$$
\begin{aligned}
& \mathrm{dPVC}=-k_{1}^{\prime} \exp \left(-E a_{1}^{\prime} / R T\right)[\mathrm{PVC}] \\
& \mathrm{dPVC}^{*}=b\left(k_{1}^{\prime} \exp \left(-E a_{1}^{\prime} / R T\right)[\mathrm{PVC}]-k_{2}^{\prime} \exp \left(-E a_{2}^{\prime} / R T\right)\left[\mathrm{PVC}^{*}\right]\right)
\end{aligned}
$$

\begin{tabular}{|c|c|c|c|c|c|c|c|c|c|}
\hline \multirow{2}{*}{$\begin{array}{l}\text { Heating rate } \\
\left({ }^{\circ} \mathrm{C} / \mathrm{min}\right)\end{array}$} & \multicolumn{3}{|c|}{ DTG peak temperature $\left({ }^{\circ} \mathrm{C}\right)$} & \multicolumn{3}{|c|}{ DTG value (\%/min) } & \multicolumn{3}{|c|}{ Weight loss $(\%)$} \\
\hline & First loss & Second loss & Third loss & First loss & Second loss & Third loss & First loss & Second loss & Third loss \\
\hline 5 & 270 & 325 & 458 & -9.5 & -1.2 & -2.2 & 56.3 & 10.9 & 25.6 \\
\hline 10 & 283 & 336 & 470 & -15.5 & -2.3 & -3.9 & 58.1 & 10 & 25.6 \\
\hline 20 & 300 & 350 & 480 & -22.3 & -6.3 & -6.5 & 58 & 10.6 & 24.4 \\
\hline 30 & 312 & - & 483 & -26.6 & - & -8.6 & 56.3 & 13.1 & 23.1 \\
\hline
\end{tabular}

Table 2

Weight losses, DTG values, and DTG peak temperatures for pure PVC

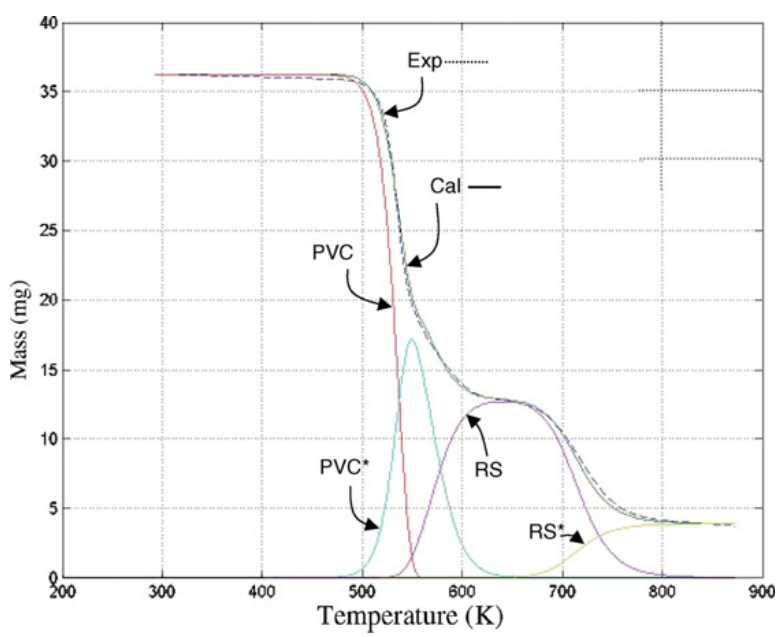

Fig. 5. Experimental and calculated curves for pure PVC. 


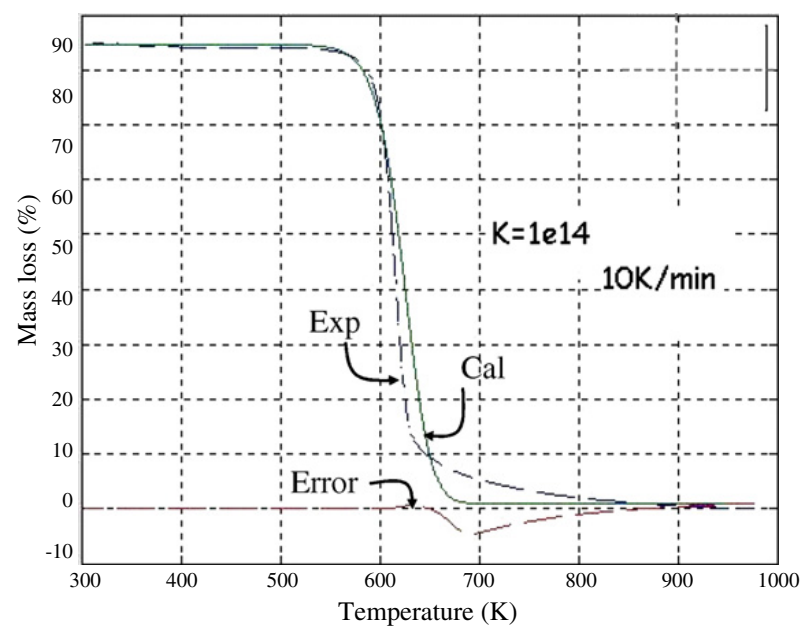

Fig. 6. Experimental and calculated curves for pure cellulose.

Table 3

Weight losses, DTG values, and DTG peak temperatures for pure cellulose

\begin{tabular}{llll}
\hline Heating rate $\left({ }^{\circ} \mathrm{C} / \mathrm{min}\right)$ & DTG peak temperature $\left({ }^{\circ} \mathrm{C}\right)$ & DTG value $(\% / \mathrm{min})$ & Weight loss $(\%)$ \\
\cline { 2 - 4 } & Weight loss & Weight loss & Weight loss \\
\hline 10 & 345 & -23.3 & 83 \\
30 & 364 & -37 & 83 \\
\hline
\end{tabular}

$$
\begin{aligned}
& \mathrm{dRS}=e\left(k_{2}^{\prime} \exp \left(-E a_{2}^{\prime} / R T\right)\left[\mathrm{PVC}^{*}\right]-k_{3}^{\prime} \exp \left(-E a_{3}^{\prime} / R T\right)[R S]\right) \\
& \mathrm{dRS}^{*}=g\left(k_{3}^{\prime} \exp \left(-E a_{3}^{\prime} / R T\right)[R S]\right) \\
& \mathrm{dPVC}_{\text {total }}=\mathrm{dPVC}+\mathrm{dPVC}^{*}+\mathrm{dRS}
\end{aligned}
$$

Fig. 4 confirms the very close agreement between the experimental results and the values simulated using the model selected for pyrolysis of PVC alone. The intermediate species and their stability zones account for the experimental curve obtained.

3.3.2.2. Cellulose. Cellulose is assumed to decompose according to two parallel reactions as shown in the following Broido-Shafizadeh reaction scheme:

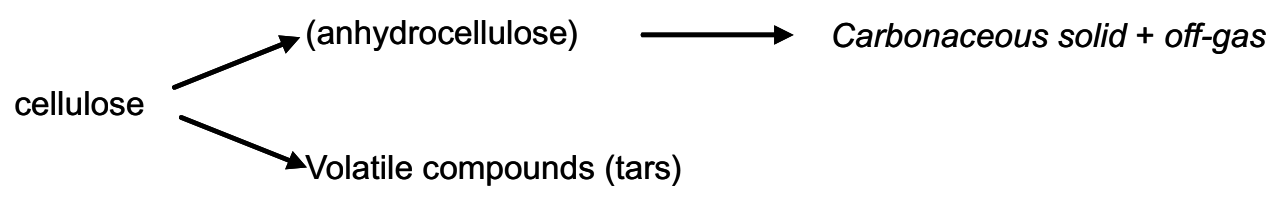

Complex kinetic models are found in the literature based on the multistage reaction mechanism described in the preceding figure. Generally, however, thermal degradation is restricted to a single step and its kinetics are described by the following simple equation $[3,4]$.

$$
\mathrm{dCellulose}=k_{0} \exp (-E / R T)(1-[\text { Cellulose }]) \cdot[\text { Cellulose }]^{0.5}
$$

The experimental approach presented in this article involves dynamic thermogravimetric analysis, with discrimination of the published kinetic equations for thermal decomposition of solids.

The reaction rate is expressed as follows:

$$
\mathrm{d} X / \mathrm{d} t=k_{0} \exp (-E / R T) \cdot f(X)
$$


Table 4

$f(X)$ functions applied to thermal degradation of solids

\begin{tabular}{ll}
\hline Model & $f(X)$ \\
\hline $\begin{array}{l}\text { Reaction order } \\
n=0\end{array}$ & $(1-X)^{n}$ \\
$n=1$ & \\
$n>1$ & \\
Geometric functions & $2(1-X)^{0.5}$ \\
Cylindrical symmetry & $3(1-X)^{2 / 3}$ \\
Spherical symmetry & \\
Diffusion functions & $1 / 2 X$ \\
1-Dimension & $(-\operatorname{Ln}(1-X))^{-1}$ \\
2-Dimensions & $1.5(1-X)^{2 / 3}\left(1-(1-X)^{1 / 3}\right)^{-1}$ \\
3-Dimensions: Jander equation & $1.5\left((1-X)^{-1 / 3}-1\right)^{-1}$ \\
3-Dimensions: Ginstling-Brounshtein & \\
Crystal growth & $n(1-X)(-\operatorname{Ln}(1-X))^{(n-1) / n}$ \\
Avrami-Erofeev for $n=1.5,2,3$ & $(1-X) X^{n}$ \\
Prout-Tomkins for $n=0.5,1$ & \\
\hline
\end{tabular}

in which $X$ represents solid conversion rate and $f(X)$ is a mathematical function depending on the presumed reaction mechanism (Table 4).

Prior studies have shown that the most suitable basis for modelling the kinetics of cellulose degradation is the Prout-Tomkins equation, which was therefore adopted for this study.

The results obtained with the model (Fig. 6) differed from the experimental values, although the model closely approximated reality except for the final stage. This deviation justifies the published corrective terms applied to the Prout-Tomkins equation.

\subsection{Discussion and conclusion}

By modelling kinetic equations using MATLAB we were able to compare our results with previously published theoretical models. The good agreement encouraged us to consider these models as valid for studying the behavior of polymers. In the case of PVC, degradation occurs in two steps separated by the appearance of an intermediate reaction product. Cellulose is decomposed directly and although the model error margin was greater, there did not appear to be any intermediate compound in the process.

The incineration test results combined with the modelling results suggest that the appearance of intermediate compounds can be responsible for the instability of the process. Additional tests that are not discussed here also suggest that phenomena similar to those observed during incineration of waste rich in PVC occur when waste is enriched in ethylene vinyl acetate (EVA), which also produces an intermediate decomposition compound during the degradation process. In this case the compound was isolated, and exhibits properties similar to those of alkanes. Being a liquid, it can impregnate carbonaceous residues such as those produced by pyrolysis of cellulose. This is why mixtures that include intermediate degradation products and large quantities of cellulose result in the formation of major deposits that significantly perturb the operation of the unit. These conclusions indicate that it would be advisable to eliminate these compounds. A chemical method appears to be difficult to implement, as it would require adding compounds that could affect the off-gas treatment system. A more appropriate solution could be to modify the process control procedures.

\section{Implementation of raw material feed sequencing in the furnace}

Fig. 5 shows that the intermediate compound degrades between 600 and $700{ }^{\circ} \mathrm{C}$ for a heating rate of $20^{\circ} \mathrm{C} \mathrm{min}{ }^{-1}$. This temperature exceeds that of the pyrolysis kiln in the IRIS process, but could be maintained for a residence time of about $5 \mathrm{~min}$. It was therefore considered advantageous to modify the furnace operation by including periodic 6 min pauses to ensure degradation of the intermediate compounds. 
The following sequence was initially proposed:

Feed: 20 min,

Pause: $6 \mathrm{~min}$.

\subsection{Including the effect of sequencing in the heat balance}

The fact that power is injected cyclically into the furnace must be taken into account in integrating expression (2) by including a periodic material feed function that can be represented by a Fourier function:

$$
D(t)=\frac{a_{0}}{2}+\sum_{n=1}^{\infty}\left(a_{n} \cos n \omega t+b_{n} \sin \omega t\right)
$$

Initially, the $a_{0}$ term is followed by a periodic function with a period $T$. Thus, as a rough approximation:

$$
D(t)=\frac{a_{0}}{2}+A \cos \omega t
$$

where

$$
a_{0}=\frac{2}{T} \int_{-\frac{T}{2}}^{+\frac{T}{2}} D(t) \mathrm{d} t
$$

For this application it is easy to calculate $a_{0}$ for a function representing a heat flow rate of $5 \mathrm{~kg} / \mathrm{h}$ for 20 min and $0 \mathrm{~kg} / \mathrm{h}$ for $6 \mathrm{~min}$ (corresponding to a mean heat flow rate of about $4 \mathrm{~kg} / \mathrm{h}$ ):

$$
a_{0}=\frac{20}{13} Q
$$

where $Q$ is the continuous feed rate. In this case, the heat balance is best described by the following differential equation:

$$
\frac{\mathrm{d}(\Delta \Theta)}{\mathrm{d} t}+\frac{K}{C} \Delta \Theta=\frac{1}{C}\left[\frac{20}{26} Q+A \cos (\omega t)\right]
$$

The general solution to this equation with its constant $20 Q / 26 C$ is given by expression (3). In seeking a particular solution to this equation with a periodic function, a factor $A$ must be considered such that

$$
\Delta \Theta=A(t) \mathrm{e}^{-\frac{K}{C} t}
$$

Hence it can be inferred that

$$
\frac{\mathrm{d} A}{\mathrm{~d} t}=\mathfrak{R} e\left[\lambda \mathrm{e}^{\left(\frac{K}{C}+\mathrm{i} \omega\right) t}\right]
$$

i.e. in complex notation:

$$
\bar{A}=\frac{\lambda}{\frac{K^{2}}{C^{2}}+\omega^{2}}\left(\frac{K}{C}-\mathrm{i} \omega\right) \mathrm{e}^{\left(\frac{K}{C}+\mathrm{i} \omega\right) t}
$$

By postulating that $\beta=\frac{K^{2}}{C^{2}}+\omega^{2}$, the general solution to expression (18) is the following:

$$
\Theta=\Theta_{0}+\frac{20}{26} \frac{Q}{K}\left(1-\mathrm{e}^{-\frac{K}{C} t}\right)+\frac{\lambda}{\beta}\left(\frac{K}{C} \cos \omega t+\omega \sin \omega t\right)
$$

Expression (19) reveals that the temperature will oscillate if coefficient $\lambda$, the reciprocal of the overall thermal damping, is too high.

\subsection{Application of sequencing to heat treatment of polymer mixtures}

Composition B (see Table 1) was processed as described in paragraph 2 except that the load was supplied to the kiln according to sequence specified above. Fig. 7 shows the temperature recorded in the pyrolysis furnace over a 4-day test period. 


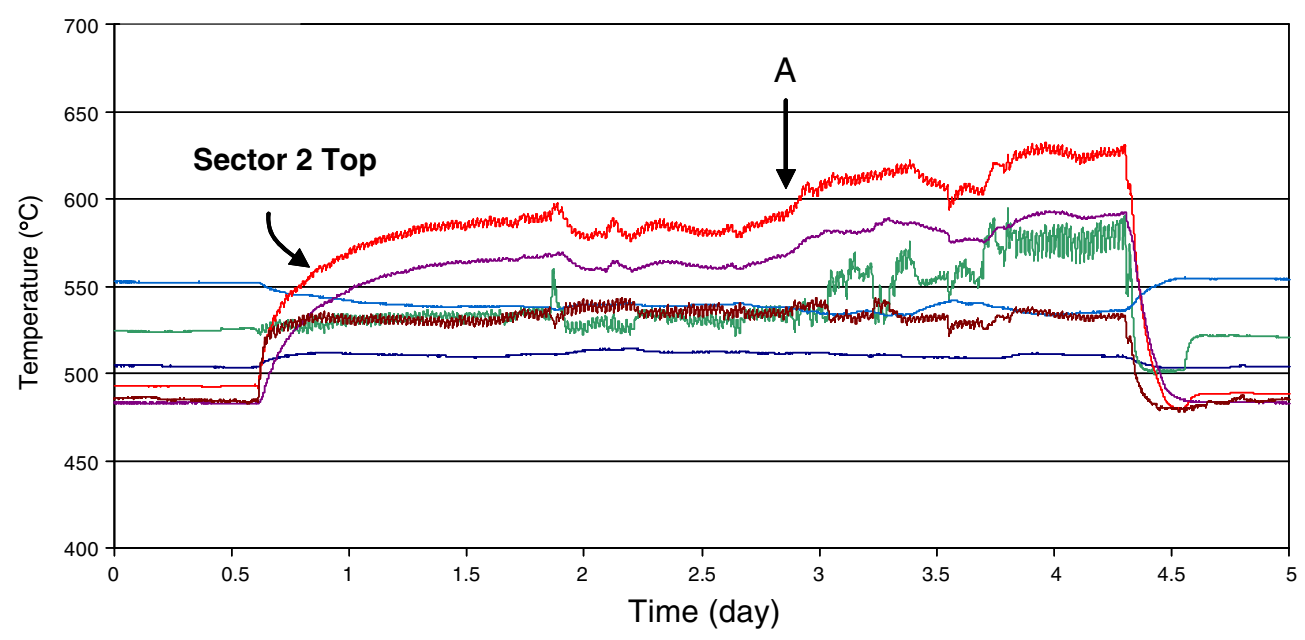

Fig. 7. Temperature variations in an oxidizing pyrolysis kiln during processing over a 2.5 -day period.

The pyrolysis furnace temperature curves (Fig. 7) show:

- excellent stability throughout the furnace (only zone 2 is referenced in Fig. 7),

- disappearance of the sudden, uncontrolled temperature deviations.

Compared with the results shown in Fig. 1, which were obtained by processing the same mixture under the same conditions (except with continuous feeding), the results obtained with sequencing are remarkable. It is interesting to note that the pressure values and pressure losses in the entire process are also controlled, making the process control much more reliable. An application to hazardous (chemical or nuclear) waste processing is thus of particular interest.

The curves obtained in zone 2 of the pyrolyzer are similar to those predicted by theoretical analysis. This is illustrated in Fig. 8 by an enlarged inset of the results obtained up to point $A$, corresponding to an increase in the feed rate. In this case, the calculated parameters are such that $K_{3} / C_{3}=5.5$ and $20 Q_{3} / 26 K_{3}=90$. Then, it seems in this case that the deposit in the furnace still exists what is confirmed by the ratio $K_{3} / C_{3}$ lower than $K_{1} / C_{1}$. But one can see that this deposit is under control and does not disturb the running of the kiln.

The inset in Fig. 8 shows that the pulsation imposed by the material feed is precisely reproduced in the temperature curve. The very good fit between the two curves also indicates excellent pyrolysis regularity.

It is interesting to note that the temperature fluctuations are found in the first zone of the afterburner [1] due to the cyclic arrival of combustible gases from the pyrolyzer.

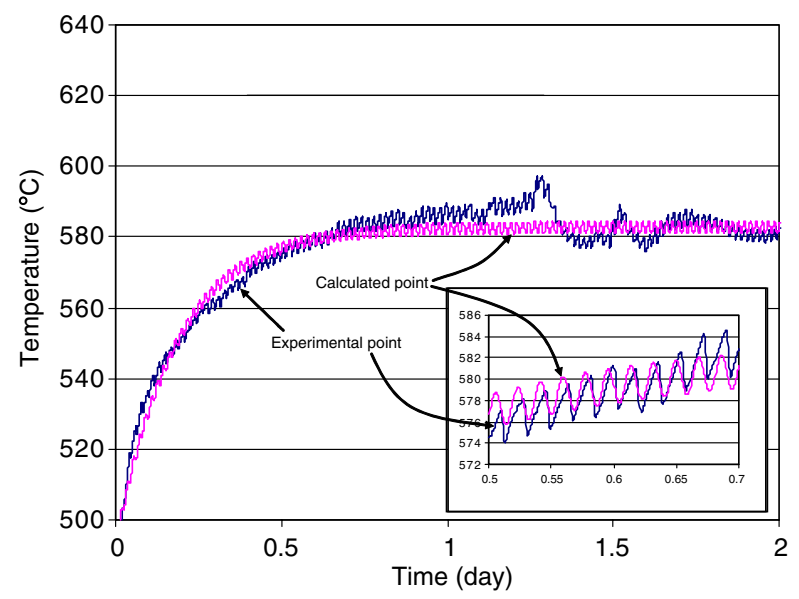

Fig. 8. Temperature measured in pyrolyzer zone 2 versus calculated temperature. 


\section{Conclusion}

Pyrolytic processing of chlorinated organic waste in a rotating kiln revealed that some waste mixtures containing a large proportion of cellulose result in major malfunctions due to deposits in the furnace. A thermogravimetric analysis of PVC degradation supported by modelling with a known degradation mechanism highlighted the appearance of intermediate reaction compounds with an appreciable lifetime. By combining with other compounds such as carbonaceous residues from pyrolytic degradation of cellulose, these compounds appear to be responsible for the difficulties encountered. Actually, even if the degradation of pure cellulose leads to give no residues as it appears in Fig. 6, the intermediate compounds coming from the degradation of other compounds such as PVC or EVA could embed the cellulose or other before they are totally pyrolyzed. Some studies are performed today in order to understand the mechanisms involved in the pyrolysis of mixtures. Sequencing the waste feed in the pyrolyzer creates a furnace relaxation period exceeding the lifetime of the intermediate compounds at the process temperature. Experimental tests implementing sequential operation significantly improved the operation of the furnace and of the entire processing facility. The temperatures and pressures at each step in the process remained very stable - an important factor in ensuring the safety and optimum quality of the process. Further investigations are currently in progress with other compounds to identify the intermediate degradation species and their interactions with other compounds that may be present during the pyrolysis step, even under slightly oxidizing conditions. Pending the availability of these results, the revised operating mode has been selected for incineration of radioactive organic waste by the IRIS process. It could also be successfully applied to processing other sensitive organic waste requiring stringent safety precautions.

\section{References}

[1] F. Lemort, J.P. Charvillat, Incineration of chlorinated organic nuclear waste: in situ substitution of phosphates for chlorides, ICEM'99 Conference, Nagoya, Japan, 26-30 September 1999.

[2] A. Marcilla, M. Beltran, Thermogravimetric kinetic study of poly(vinyl chloride) pyrolysis, Polymer Degradation and Stability 48 (1995) 219-229.

[3] Y. Matsuzawa, M. Ayabe, J. Nishino, Acceleration of cellulose co-pyrolysis with polymer, Polymer Degradation and Stability (2001) 435-444.

[4] L. Khezami, R. Carpart, 9e Congrès SFGP St Nazaire, France, 9-11 September 2003. 